\title{
Predicting the unpredictable: is it possible clinically to separate H1N1 from non-H1N1 community- acquired pneumonia?
}

\author{
Nita Sehgal, Mark Woodhead
}

In the spring of 2009 a novel influenza A virus (H1N1) of swine origin was identified in the USA and Mexico and rapid spread led to the declaration of a global influenza pandemic by the World Health Organization in June 2009. ${ }^{12}$ The virus, which derives six genes from triplereassortant North American swine virus lineages and two genes from Eurasian swine virus lineages, predominantly affected children and young adults with a low incidence of acute illness in those over 60 years of age. ${ }^{3}$ By June 2010, cases had been identified in over 200 countries and territories worldwide and over 18000 associated deaths were reported. ${ }^{4}$

Infection with the pandemic virus caused a broad spectrum of clinical disease ranging from acute self-limiting upper respiratory tract illness with fever to fulminant pneumonitis and respiratory failure. Gastrointestinal and CNS symptoms were also common. Rates of admission to ICUs $(13-45.3 \%$ of patients being hospitalised) were much higher than previously seen with seasonal influenza. ${ }^{5-8}$ Rapid progression of severe hypoxaemia, typically starting on day $4-5$ after the onset of symptoms, was observed with up to $80 \%$ of patients admitted to an ICU requiring mechanical ventilation. ${ }^{6}$ Frequent radiological findings were of diffuse alveolar and interstitial infiltrates, often worse in the lower zones.

A number of risk factors were identified for the development of severe disease including age $<2$ years, pregnancy (highest risk in the third trimester), immunosuppression, morbid obesity, chronic cardiovascular and respiratory disease, diabetes and neuromuscular and neurocognitive

Department of Respiratory Medicine, Manchester Royal Infirmary, Manchester, UK

Correspondence to Mark Woodhead, Department of Respiratory Medicine, Manchester Royal Infirmary, Oxford Road, Manchester M13 9WL, UK;

mark.woodhead@cmft.nhs.uk conditions. The lowest rates of infection were seen in those aged >65 years, presumably relating to immunity conferred from prior exposure to antigenically-related $\mathrm{H} 1 \mathrm{~N} 1$ virus in previous outbreaks. Paradoxically, death rates were highest in this group. ${ }^{3}$

By August 2010 the World Health Organization announced that the H1N1 influenza virus had moved into the postpandemic period but warned that the 2009 H1N1 virus was expected to circulate as a seasonal virus based on knowledge from past pandemics. This has certainly been the case in the UK where laboratory-confirmed cases have soared. By 24 December 2010, 460 critical care beds in the UK were occupied with patients with $\mathrm{H} 1 \mathrm{~N} 1$ infection and the latest figures from 12 January 2011 confirm a total of 112 influenza-related deaths in the UK with $85 \%$ of these occurring in patients with 2009 H1N1 virus. 9 Respiratory sequelae and pneumonia remain the commonest precipitating factor for hospitalisation and critical care admissions.

While the balance between the benefits and harms of treatment with neuraminidase inhibitors (NIs) in H1N1 infection in previously healthy adults remains uncertain, ${ }^{10}$ they are now accepted as a standard of care for patients with H1N1-related pneumonia. ${ }^{11}$ NIs are recommended for all patients with complicated illness (defined as those with an influenza-like illness requiring hospital admission and/or presenting with symptoms and signs of lower respiratory tract infection, CNS involvement or other form of target organ damage and/or exacerbation of an underlying condition) or in those with a progressive illness or highrisk individuals. ${ }^{12}$ It is advocated that treatment is started empirically as early as possible. The early identification of appropriate patients for such treatment may thus be important. Conversely, while isolation policies are recommended for patients with $\mathrm{H} 1 \mathrm{~N} 1$ infection, correct triage can limit the pressures on this finite resource. So, can H1N1-related pneumonia be identified early?

In this issue of Thorax Bewick et al report that adults admitted to hospital with pandemic $\mathrm{H} 1 \mathrm{~N} 1$ influenza-related pneumonia can be distinguished from patients with non-H1N1 pneumonia based on clinical and laboratory findings (see page 247)..$^{13}$ During 8 months of the pandemic period, the Influenza Clinical Information Network identified 1046 adults admitted to UK hospitals with confirmed H1N1 infection by PCR testing and, of these, $25 \%$ were found to have radiographic changes consistent with a diagnosis of pneumonia. This cohort was compared with 648 patients with inter-pandemic community-acquired pneumonia admitted to a single centre during a period in which $\mathrm{H} 1 \mathrm{~N} 1$ influenza circulation was deemed negligible in the study catchment area. Despite having similar in-hospital mortality, there was a striking difference between the two groups in terms of age (median age 42 years in the H1N1 cohort vs 75 years in the non-H1N1 cohort), comorbidities and critical care admissions $(34.6 \%$ in the $\mathrm{H} 1 \mathrm{~N} 1$ cohort vs $12.8 \%$ in the non-H1N1 cohort). In a logistic regression model, five categorical variables at the time of admission were found to be associated with H1N1-related pneumonia and a diagnostic prediction model was derived based on age $\leq 65$ years, presence of bilateral radiographic consolidation, absence of confusion, leucocyte count $\leq 12 \times 10^{9} / 1$ and temperature $\geq 38^{\circ} \mathrm{C}$. Assigning a point for each of these clinical criteria enabled patients to be stratified into three risk groups. Only one of 226 $(0.4 \%)$ with a score of 0 or 1 had H1N1 influenza-related pneumonia while 126 of $168(75 \%)$ with scores of 4 or 5 had such infection.

In order for this model to provide clinicians with a simple tool for directing therapy, it is essential that the patients studied in the influenza cohort are representative of patients being hospitalised with a pneumonic illness. Bewick et al identified 254 patients with H1N1 influenza-related pneumonia across 75 UK hospitals, giving an average enrolment rate of only 3.4 cases per site over 8 months. Patients were tested for H1N1 influenza on the basis of clinical suspicion-significant numbers may therefore have been missed with a potential case selection bias towards previously recognised clinical phenotypes. The ICU 
admission rate is at the higher end of the range reported in other studies, ${ }^{8} 14$ perhaps supporting potential bias towards more severe cases.

In addition, PCR testing was only performed on samples obtained via a nasopharyngeal swab or bronchoalveolar lavage. Evidence suggests that the yield of positive PCR results from nasopharyngeal swabs in patients with confirmed positive lower respiratory tract samples is between $63 \%$ and $81 \%{ }^{15}{ }^{16}$ World Health Organisation guidance recommends that a negative result in single respiratory specimens does not rule out 2009 H1N1 virus infection and advocates repeated sampling when clinical suspicion is high. ${ }^{3}$ Patients with $\mathrm{H} 1 \mathrm{~N} 1$ related-influenza may therefore have been excluded on the basis of one false negative upper airway specimen.

Despite these limitations, the conclusion by Bewick et al that H1N1 influenza pneumonia differs substantially from interpandemic community-acquired pneumonia is supported by their findings and is probably correct. Whether the differences observed are clinically useful is more difficult to determine. As a predictor of who should receive a NI, the findings might be useful in an influenza outbreak where H1N1 is the only cause, as in the pandemic. However, in seasonal outbreaks, infection is often a result of more than one circulating virus. This is seen in the current UK outbreak which, while predominantly due to $2009 \mathrm{H} 1 \mathrm{N1}$, is also caused by influenza A H3N2 and influenza B which can cause severe illness meriting NI treatment. Indeed, in the week ending 6 January 2011, the HPA had identified up to $25 \%$ of influenza isolates in England as influenza B. ${ }^{9}$ In addition, the findings only apply to younger adults who predominantly made up the cohort of $\mathrm{H} 1 \mathrm{~N} 1$ pneumonia cases included in the study. The findings may not apply to children who have a very high incidence of infection or to other subgroups in which the clinical spectrum may be different, such as patients with cystic fibrosis (see letters by Colombo and Nash in this issue of Thorax (see pages 260, 259)). ${ }^{17} 18$ The virus is subject to constant antigenic change, so the clinical picture with future variants of the virus may not be the same and different age groups may be predominantly affected.

Rather than identifying patients who might benefit from NI treatment, the most useful finding of the model proposed by Bewick et al is perhaps the high negative predictive value of a score of 0 or 1 . Inpatient isolation and NI treatment might safely be avoided in these patients if they are adults aged $<65$ years. However, before recommending this approach in practice, further validation is required ideally in a larger study with contemporary collection of cases and controls using rigorous criteria for $\mathrm{H} 1 \mathrm{~N} 1$ testing in both groups.

\section{Competing interests None.}

Provenance and peer review Commissioned; not externally peer reviewed.

Thorax 2011;66:187-188.

doi:10.1136/thx.2010.157404

\section{REFERENCES}

1. Novel Swine-Origin Influenza A (H1N1) Virus Investigation Team. Emergence of a novel swineorigin influenza $\mathrm{A}(\mathrm{H} 1 \mathrm{~N} 1)$ virus in humans. N Engl J Med 2009; 360:2605-15.

2. Perez-Padilla R, Rosa-Zamborini D, Ponce de Leon $S$, et al. Pneumonia and respiratory failure from swineorigin influenza A (H1N1) in Mexico. N Engl J Med 2009;361:680-9.

3. Writing Committee of the WHO Consultation on Clinical Aspects of Pandemic (H1N1) 2009 Influenza. Clinical aspects of pandemic 2009 influenza A (H1N1) virus infection. N Engl J Med 2010;362:1708-19.

4. World Health Organization. Pandemic (H1N1) 2009. 2009. http://www.who.int/csr/don/2010 06 11/en/index.html.
5. Jain S, Kamimoto L, Bramley AM, et al. Hospitalized patients with 2009 H1N1 influenza in the United States, April-June 2009. N Engl J Med 2009:361:1935-44.

6. Ramsey C, Kumar A. H1N1: viral pneumonia as a cause of acute respiratory distress syndrome. Curr Opin Crit Care 2011;17:64-71.

7. Riquelme R, Torres A. Influenza pneumonia: a comparison between seasonal influenza virus and H1N1 pandemic. Eur Respir J Published Online First: 25 Nov 2010. doi:10.1183/09031936.00125910.

8. Falagas ME, Koletsi PK, Baskouta E, et al. Pandemic A (H1N1) 2009 influenza: review of the Southern Hemisphere experience. Epidemiol Infect 2011:139:27-40.

9. McLean E, Pebody R. HPA Weekly National Influenza Report: Summary of UK Surveillance of Influenza and Other Respiratory IIIness. 12.0.11.

10. Jefferson T, Jones MA, Di Pietrantonj C, et al. Neuraminidase inhibitors for preventing and treating influenza in healthy adults. Cochrane Database Syst Rev 2006(3): CD001265.

11. National Institute for Health and Clinical Excellence (NICE). Amantadine, Oseltamivir Zanamivir for the Treatment of Influenza: Review of NICE Technology Appraisal Guidance 58. London: NICE, 2009 .

12. Health Protection Agency. Pharmacological Treatment and Prophylaxis of Influenza. Health Protection Agency, 2011. Version 1.72011.

13. Bewick T, Myles P, Greenwood S, et al. Clinical and laboratory features distinguishing pandemic H1N1 influenza-related pneumonia from interpandemic community-acquired pneumonia in adults. Thorax 2011:66:247-52.

14. Helferty M, Vachon J, Tarasuk J, et al. Incidence of hospital admissions and severe outcomes during the first and second waves of pandemic (H1N1) 2009. CMAJ 2010;182:1981-7.

15. Blyth C, Iredall J, Dwyer D. Rapid-test sensitivity for novel swine-origin influenza A (H1N1) virus in humans. N Engl J Med 2009; 361:2493

16. Mulrennan S, Tempone SS, Wai Ling IT, et al. Pandemic influenza (H1N1) 2009 pneumonia: CURB65 score for predicting severity and nasopharyngeal sampling for diagnosis are unreliable. PLoS One 2010;5:e12849.

17. Colombo C, Battezzati PM, Lucidi V, et al. Influenza A/H1N1 in patients with cystic fibrosis in Italy: a multicentre cohort study. Thorax 2011;66:260-1.

18. Nash EF, Whitmill R, Barker B, et al. Clinical outcomes of pandemic (H1N1) 2009 influenza (swine flu) in adults with cystic fibrosis. Thorax 2011:66:259. 